\title{
An investigation on the Lasota-Wazewska model with a piecewise constant argument
}

\author{
Gizem S. Oztepe \\ Department of Mathematics, Ankara University, 06100, Ankara, Turkey
}

\begin{abstract}
This paper is devoted to investigating the asymptotic stability of the equilibrium point of the Lasota-Wazewska model with a piecewise constant argument and it is proved that this point is an attractor. It is also shown that every oscillatory solution of the corresponding difference equation has semi-cycles of length at least two.
\end{abstract}

Mathematics Subject Classification (2020). 34K09, 34K11, 34K20, 39A30

Keywords. Lasota-Wazewska model, piecewise constant argument, asymptotic stability, oscillation

\section{Introduction}

The aim of this paper is to investigate the stability and oscillation properties of the positive solutions of the Lasota-Wazewska model with a piecewise constant argument

$$
x^{\prime}(t)=-\alpha x(t)+\beta e^{-\gamma x(\lfloor t-1\rfloor)},
$$

where $\alpha, \beta$ and $\gamma$ are positive constants and $\lfloor$.$\rfloor denotes the floor function which gives the$ greatest integer less than or equal to given number. Equation (1.1) is generated from the delay differential equation

$$
x^{\prime}(t)=-\alpha x(t)+\beta e^{-\gamma x(t-\tau)}
$$

which was first used as a mathematical model for the survival of red blood cells in an animal by Wazewska-Czyzewska and Lasota [62]. Here $x(t)$ denotes the number of red blood cells at time $t, \alpha>0$ is the probability of death of a red blood cell, $\beta>0$ is the constant connected with demand for oxygen, $\gamma$ is also a positive constant characterizes excitability of haematopoietic system and $\tau$ is the time required to produce a red blood cell. Further, we refer to [34] for biological models, involving chemotaxis and nonlinear diffusive mechanism, formulated by the introduction of reactions coupling growth and death impacts. The well known Lasota-Wazewska model (1.2) was extended and generalized by many authors, see for example $[3,19,22,23,29,30,32,33,43,65]$ including some recent publications on the topic $[8,11,14,26,52,54,56,64]$.

On the other hand, Eq. (1.1) is called a differential equation with a piecewise constant argument [6], [63]. These type of equations have attracted great deal of attention because

Email address: gseyhan@ankara.edu.tr

Received: 08.07.2020; Accepted: 07.06.2021 
they combine the properties of both continuous and discrete dynamical systems. In mathematical modelling considering both continuous and discrete times makes sense so the use of piecewise constant arguments come into question, see $[2,4,7,13,21,25,42,50,55,61]$ and the references therein. To the best of our knowledge the Lasota-Wazewska model with a piecewise constant argument was considered in [9], [12] and [51]. In [9], it was shown that Eq. (1.1) has a unique almost automorphic solution, in [12] Eq. (1.1) with generalized piecewise constant argument was considered and the existence of periodic solutions of this equation was proved and in [51], the existence and global attractivity condition of almost periodic solution and quasi-periodic solution of Eq. (1.1) were obtained. Moreover, discrete version of the Lasota-Wazewska model was studied in many papers $[5,10,44,53,58]$.

Besides, global attractivity and stability are also among the most popular subjects in the study of both difference equations and equations with piecewise constant arguments $[15,17,18,21,24,27,35,41,45-49,59,60]$. Another current issue used in the study of difference equations is the semi-cycle analysis, which was initiated by [28] and then attracted great attention in different fields of the difference equations [1,20,36-40,57].

This paper is organized as follows. In Section 2 basic definitions and some useful well known results are stated. In Section 3, the main results of the paper are given with some examples.

\section{Preliminaries}

In this section we will introduce a solution of Eq. (1.1) and give some results that we will use in the next section.

Definition 2.1. A function $x(t)$ defined on the set $\{-1\} \cup[0, \infty)$ is called a solution of Eq. (1.1) if it satisfies the following conditions:

(1) $x(t)$ is continuous on $[0, \infty)$,

(2) $x(t)$ is differentiable and for any $t \in[0, \infty)$ it satisfies Eq. (1.1) with the possible exception of the points $\lfloor t\rfloor$ in $[0, \infty)$ where one sided derivatives exist.

Since Eq. (1.1) has a biological meaning, we only take into account the positive solutions. For this purpose we consider Eq. (1.1) with the initial conditions

$$
x(-1)=x_{-1}>0, \quad x(0)=x_{0}>0 .
$$

Let $I$ be some interval of real numbers and let $f \in C^{1}(I \times I, I)$. Let $x^{*} \in I$ be an equilibrium point of the difference equation

$$
x_{n+1}=f\left(x_{n}, x_{n-1}\right), \quad n=0,1,2, \ldots
$$

Let

$$
p=\frac{\partial f}{\partial u}\left(x^{*}, x^{*}\right) \quad \text { and } \quad q=\frac{\partial f}{\partial v}\left(x^{*}, x^{*}\right)
$$

denote the partial derivatives of $f(u, v)$ evaluated at an equilibrium $x^{*}$ of Eq. (2.2). Then the equation

$$
y_{n+1}=p y_{n}+q y_{n-1}, \quad n=0,1,2, \ldots
$$

is called the linearized equation associated with Eq. (2.2) about the equilibrium point $x^{*}$.

Theorem 2.2 (Linearized stability, Theorem A in [16]).

(1) If both roots of the quadratic equation

$$
\lambda^{2}-p \lambda-q=0
$$

lie in the open disk $|\lambda|<1$, then the equilibrium $x^{*}$ of Eq. (2.2) is locally asymptotically stable.

(2) If at least one of the roots of Eq. (2.4) has absolute value greater than one, then the equilibrium $x^{*}$ of $E q .(2.2)$ is unstable. 
(3) A necessary and sufficient condition for both roots of Eq. (2.4) to lie in the open unit disk $|\lambda|<1$, is

$$
|p|<1-q<2,
$$

in this case the locally asymptotically stable equilibrium $x^{*}$ is also called a sink.

Semi-cycle analysis of the solutions of the scalar difference equation is thought to be a powerful tool for a thorough understanding of the entire character of solutions. Now let's give the definitions of positive and negative semi-cycle of a solution of Eq. (2.2) relative to an equilibrium point $x^{*}$.

Definition 2.3 ([31, pages 24-25]). A positive semi-cycle of a solution $\left\{x_{n}\right\}$ of Eq. (2.2) consists of a "string" of terms $\left\{x_{l}, x_{l+1}, \ldots, x_{m}\right\}$, all greater than or equal to equilibrium $x^{*}$, with $l \geq-1$ and $m \leq \infty$ such that

$$
\text { either } \quad l=-1 \quad \text { or } \quad l>-1 \quad \text { and } \quad x_{l-1}<x^{*}
$$

and

$$
\text { either } m=\infty \text { or } m<\infty \text { and } x_{m+1}<x^{*} \text {. }
$$

A negative semi-cycle of a solution $\left\{x_{n}\right\}$ of Eq. (2.2) consists of a "string" of terms $\left\{x_{l}, x_{l+1}, \ldots, x_{m}\right\}$, all less than the equilibrium $x^{*}$, with $l \geq-1$ and $m \leq \infty$ such that

$$
\text { either } \quad l=-1 \quad \text { or } \quad l>-1 \quad \text { and } \quad x_{l-1} \geq x^{*}
$$

and

$$
\text { either } m=\infty \text { or } m<\infty \text { and } x_{m+1} \geq x^{*} .
$$

Theorem 2.4 ([31, Theorem 1.7.4]). Assume that $f \in C[(0, \infty) \times(0, \infty),(0, \infty)]$ is such that $f(x, y)$ is increasing in $x$ for each fixed $y$ and $f(x, y)$ is decreasing in $y$ for each fixed $x$. Let $x^{*}$ be a positive equilibrium of Eq. (2.2). Then except possibly for the first semi-cycle, every oscillatory solution of Eq. (2.2) has semi-cycles of length at least two.

Theorem 2.5 ([28, Theorem 2.4.1]). Consider the difference equation

$$
x_{n+1}=a x_{n}+F\left(x_{n-k}\right), n=0,1, \ldots
$$

where

$$
a \in[0,1), \quad k \in\{1,2, \ldots\} \quad \text { and } \quad F \in C[[0, \infty),(0, \infty)] .
$$

Assume that (2.7) holds. $F(u)$ is decreasing in $u$, and that the system

$$
U=\frac{F(L)}{1-a} \quad \text { and } \quad L=\frac{F(U)}{1-a}
$$

has exactly one solution $\{L, U\}$ in the positive quadrant $(0, \infty) \times(0, \infty)$. Then Eq. (2.6) has a unique positive equilibrium $x^{*}$. Furthermore $U=L=x^{*}$ and every solution $\left\{x_{n}\right\}$ of Eq. (2.6) with positive initial conditions

$$
x_{n}>0 \quad \text { for } \quad n=-k, \ldots, 0
$$

is attracted to $x^{*}$, that is,

$$
\lim _{n \rightarrow \infty} x_{n}=x^{*}
$$

\section{Main results}

This section devoted to find the solution of Eq. (1.1) in terms of the corresponding difference equation and to study the behaviour of these solutions.

Theorem 3.1. Eq. (1.1) with the initial conditions (2.1) has a unique solution on $\{-1\} \cup$ $[0, \infty)$. 
Proof. Let $x_{n}(t) \equiv x(t)$ be a solution of Eq. (1.1) for $t \in[n, n+1)$. Then Eq. (1.1) reduces to a linear differential equation

$$
x^{\prime}(t)+\alpha x(t)=\beta e^{-\gamma x(n-1)}, \quad n \leq t<n+1 .
$$

The solution of Eq. (3.1) is obtained as

$$
x_{n}(t)=\frac{\beta}{\alpha} e^{-\gamma x_{n-1}}+\left(x_{n}-\frac{\beta}{\alpha} e^{-\gamma x_{n-1}}\right) e^{-\alpha(t-n)}, \quad n \leq t<n+1 .
$$

On the other hand, the solution of Eq. (1.1) on the interval $[n-1, n)$, say $x_{n-1}(t)$, can be also obtained from (3.2) by considering $n-1$ in place of $n$. Then, using the continuity of the solutions leads us the nonlinear difference equation

$$
x_{n+1}=e^{-\alpha} x_{n}+\frac{\beta}{\alpha}\left(1-e^{-\alpha}\right) e^{-\gamma x_{n-1}}, \quad n=0,1,2, \ldots .
$$

Considering the initial conditions (2.1), the unique solution of Eq. (3.3) can be obtained. Hence, it is proved that the initial value problem (1.1), (2.1) has a unique solution.

Clearly, the dynamic behaviour of the model (1.1) can be determined by the difference model (3.3) because the model (3.3) is the difference solution of the model (1.1). Next we will focus on the behaviour of the difference model (3.3). For this reason first we need to find the positive equilibrium of (3.3):

Computations give us that the positive equilibrium (3.3) satisfies the equation

$$
x^{*}=\frac{\beta}{\alpha} e^{-\gamma x^{*}}
$$

which is also the critical point of Eq. (1.1).

The change of variables $z_{n}=x_{n}-x^{*}$ in Eq. (3.3) gives us the difference equation

$$
z_{n+1}=e^{-\alpha} z_{n}+\frac{\beta}{\alpha}\left(1-e^{-\alpha}\right) e^{-\gamma x^{*}} e^{-\gamma z_{n-1}}-x^{*}+x^{*} e^{-\alpha}, \quad n=0,1,2, \ldots
$$

whose equilibrium point $z^{*}=0$ corresponds to $x^{*}$.

Theorem 3.2. The equilibrium $x^{*}$ given by (3.4) is a sink if and only if

$$
\alpha e^{-\alpha}<\alpha+\beta \gamma\left(1-e^{-\alpha}\right) e^{-\gamma x^{*}}<2 \alpha .
$$

Proof. The corresponding linearized equation of Eq. (3.5) about $z^{*}=0$ is

$$
y_{n+1}=p y_{n}+q y_{n-1}, \quad n=0,1,2, \ldots
$$

where

$$
p=e^{-\alpha} \quad \text { and } \quad q=\frac{\beta \gamma}{\alpha}\left(e^{-\alpha}-1\right) e^{-\gamma x^{*}} .
$$

By applying Theorem 2.2, it is obtained that $z^{*}=0$, that is, $x^{*}$ is a sink if and only if the condition (3.6) is true.

Theorem 3.3. Every oscillatory solution of Eq. (3.3) has semi-cycles of length at least two.

Proof. We re-write the right hand side of Eq. (3.3) as

$$
f(x, y)=e^{-\alpha} x+\frac{\beta}{\alpha}\left(1-e^{-\alpha}\right) e^{-\gamma y} .
$$

The first derivatives of (3.9) with respect to $x$ and $y$ are

$$
\frac{\partial f}{\partial x}=e^{-\alpha} \quad \text { and } \quad \frac{\partial f}{\partial y}=\frac{\beta \gamma}{\alpha}\left(e^{-\alpha}-1\right) e^{-\gamma y}
$$

which are positive and negative, respectively for each $\alpha, \beta, \gamma$. So, we conclude that $f$ is increasing in $x$ and decreasing in $y$ and by Theorem 2.4, every oscillatory solution of Eq. (3.3) has semi-cycles of length at least two. 
Theorem 3.4. Every solution of Eq. (3.3) is attracted to the equilibrium $x^{*}$.

Proof. Let us apply Theorem 2.5 in our equation (3.3) under the conditions (2.1). For $k=1$ Eq. (3.3) is in the form of Eq. (2.6) with

$$
a=e^{-\alpha} \in[0,1) \quad \text { and } \quad F(u)=\frac{\beta}{\alpha}\left(1-e^{-\alpha}\right) e^{-\gamma u} .
$$

Since

$$
F_{u}=\frac{\beta \gamma}{\alpha}\left(e^{-\alpha}-1\right) e^{-\gamma u}
$$

is negative for all positive constants $\alpha, \beta$ and $\gamma$, the function $F(u)$ is decreasing. Besides, the system (2.8) can be written as

$$
\begin{aligned}
U & =\frac{\frac{\beta}{\alpha}\left(1-e^{-\alpha}\right) e^{-\gamma L}}{1-e^{-\alpha}}, \\
L & =\frac{\frac{\beta}{\alpha}\left(1-e^{-\alpha}\right) e^{-\gamma U}}{1-e^{-\alpha}} .
\end{aligned}
$$

It is easily seen that this system has exactly one solution $\{L, U\}$. So every solution of Eq. (3.3) is attracted to $x^{*}$.

Now let us give an example to illustrate our results:

Example 3.5. Let us consider equation

$$
x^{\prime}(t)=-0.1 x(t)+0.3 e^{6-2 x(\lfloor t-1\rfloor)},
$$

with the initial conditions

$$
x(-1)=1, \quad x(0)=1 .
$$

The corresponding difference equation's linearized form is

$$
y_{n+1}=0.904837 y_{n}-0.570995 y_{n-1}, \quad n=0,1,2, \ldots .
$$

Here, it can be obtained that (3.6) is true. Therefore, from Theorem 3.2, we conclude that the equilibrium point 0 of the given equation is a sink. This can be seen in Figure 1.

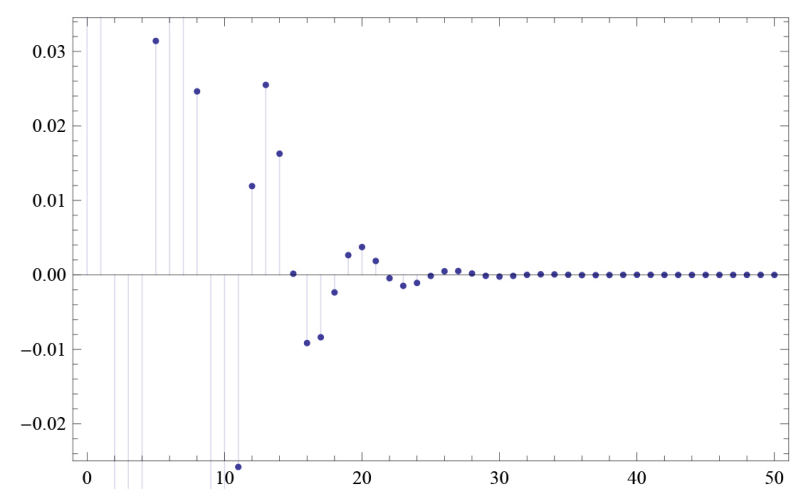

Figure 1. The behaviour of the equilibrium zero. 


\section{Conclusions}

Differential equations are very useful in understanding the real life problems since they give the mathematical expression of the real phenomena. On the other hand, modelling with ordinary differential equations is often not enough. Because while problems are mathematically established by ignoring discontinuous effects, the results of the models are far from reality. This necessity provided the introduction and development of the theory of differential equations with discontinuities. One type of equation developed as a result of this requirement is the differential equations with piecewise constant arguments. In this paper, we study the Lasota-Wazewska model to an equation with piecewise constant arguments. We investigate some qualitative properties of the corresponding difference equation which is non-linear and contains interesting properties. The method constructed in this paper can be applied to different models with piecewise constant arguments such as Nicholson blowflies, Gilpin Ayala model, predatorprey like model, Tumor-Immune interaction, tumor growth model, epidemic model, neural networks and some Volterra type models.

Acknowledgment. I am indebted to the referees for their kind and careful reading of the manuscript and for all the comments and suggestions that improved the paper considerably.

\section{References}

[1] S. Abualrub and M. Aloqeili, Dynamics of the system of difference equations $x_{n+1}=$ $A+\frac{y_{n-k}}{y_{n}}, y_{n+1}=B+\frac{x_{n-k}}{x_{n}}$, Qual. Theory Dyn. Syst. 19, 69, 2020.

[2] M. Akhmet, D.A. Çinçin and N. Cengiz, Exponential stability of periodic solutions of recurrent neural networks with functional dependence on piecewise constant argument, Turkish J. Math. 42 (1), 272-292, 2018.

[3] O. Arino and M. Kimmel, Stability analysis of models of cell production systems, Math. Modelling 7, 1269-1300, 1986.

[4] F. Bozkurt and A. Yousef, NeimarkSacker bifurcation of a chemotherapy treatment of glioblastoma multiform (GBM), Adv. Differ. Equ. 2019 (1), 397, 2019.

[5] E. Braverman and S.H. Saker, On a difference equation with exponentially decreasing nonlinearity, Discrete Dyn. Nat. Soc., 2011 1-17, 2011.

[6] L.A.V. Carvalho and K.L. Cooke, A nonlinear equation with piecewise continuous argument, Differential Integral Equations, 1 (3), 359-367, 1988.

[7] F. Cavalli and A. Naimzada, A multiscale time model with piecewise constant argument for a boundedly rational monopolist, J. Diff. Eq. App. 22 (10), 1480-1489, 2016.

[8] G.E. Chatzarakis and T. Li, Oscillation criteria for delay and advanced differential equations with nonmonotone arguments, Complexity, 2018, Article ID 8237634, 1-18, 2018.

[9] A. Chávez, S. Castillo and M. Pinto, Discontinuous almost periodic type functions, almost automorphy of solutions of differential equations with discontinuous delay and applications, Electron. J. Qual. Theory Differ. Equ. 2014 (75), 1-17, 2014.

[10] L. Chen and F. Chen, Positive periodic solution of the discrete Lasota-Wazewska model with impulse, J. Difference Equ. Appl. 20 (3), 406-412, 2014.

[11] F. Cherif and M. Miraoui, New results for a Lasota Wazewska model, Int. J. Biomath. 12 (2), 1950019, 2019.

[12] K.S. Chiu and M. Pinto, Periodic solutions of differential equations with a general piecewise constant argument and applications, Electron. J. Qual. Theory Differ. Equ. 46, 1-19, 2010. 
[13] K.S. Chiu and T. Li, Oscillatory and periodic solutions of differential equations with piecewise constant generalized mixed arguments, Math. Nachr. 292, (10), 2153-2164, 2019.

[14] L. Duan, L. Huang and Y. Chen, Global exponential stability of periodic solutions to a delay LasotaWazewska model with discontinuous harvesting, Proc. Amer. Math. Soc. 144, 561-573, 2016.

[15] J. Dudás and T. Krisztin, Global stability for the three-dimensional logistic map, Nonlinearity 34 (2), 894, 2021.

[16] M.M. El-Afifi, On the recursive sequence $x_{n+1}=\frac{\alpha+\beta x_{n}+\gamma x_{n-1}}{B x_{n}+C x_{n-1}}$, Appl. Math. Comput. 147, 617-628, 2004.

[17] Z. Feng, X. Wu and L. Yang, Stability of a mathematical model with piecewise constant arguments for tumor-immune interaction under drug therapy, Internat. J. Bifur. Chaos, 29 (01), 1950009, 2019.

[18] K. Gopalsamy, M.R.S. Kulenovic and G. Ladas, On a logistic equation with piecewise constant arguments, Differ. Int. Eq. 4, 215-223, 1991.

[19] J. Graef, C. Qian and P. Spikes, Oscillation and global attractivity in a periodic delay equation, Canad. Math. Bull. 39 (3), 275-283, 1996.

[20] M. Gumus, The global asymptotic stability of a system of difference equations, J. Difference Equ. Appl. 24 (6), 976-991, 2018.

[21] F. Gurcan and F. Bozkurt, Global stability in a population model with piecewise constant arguments, J. Math. Anal. Appl. 360 (1), 334-342, 2009.

[22] I. Györi and G. Ladas, Oscillation Theory of Delay Differential Equations with Applications, Oxford University Press, 1991.

[23] I. Györi and S.I. Trofimchuk, Global attractivity in $x^{\prime}(t)=-\delta x(t)+p f(x(t-\tau))$, Dynam. Systems Appl. 8 (2), 197-210, 1999.

[24] W.H. Joseph and J.S. Yu, Global stability in a logistic equation with piecewise constant arguments, Hokkaido Math. J. 24 (2), 269-286, 1995.

[25] F. Karakoç, Asymptotic behaviour of a population model with piecewise constant argument, Appl. Math. Lett. 70, 7-13, 2017.

[26] F. Karakoç, Asymptotic behavior of a Lasota-Wazewska model under impulse effect, Dynam. Systems Appl. 29 (12), 3381-3394, 2020.

[27] S. Kartal and F. Gurcan, Stability and bifurcations analysis of a competition model with piecewise constant arguments, Math. Methods Appl. Sci. 38 (9), 1855-1866, 2015.

[28] V.L. Kocic and G. Ladas, Global behavior of nonlinear difference equations of higher order with applications Vol. 256, Springer Science and Business Media, 1993.

[29] M.R.S. Kulenovi and G. Ladas, Linearized oscillations in population dynamics, Bull. Math. Biol. 49 (5), 615-627, 1987.

[30] M.R.S. Kulenovi and G. Ladas, Dynamics of Second Order Rational Difference Equations, Chapman and Hall/CRC, Boca Raton, 2001.

[31] M.R.S. Kulenovi, G. Ladas and Y.G. Sficas, Global attractivity in population dynamics, Comput. Math. Appl. 18 (10-11), 925-928, 1989.

[32] A. Lasota, K. Loskot and M.C. Mackey, Stability properties of proliferatively coupled cell replication models, Acta Biotheor. 39, 1-14, 1991.

[33] A. Lasota and M.C. Mackey, Cell division and the stability of cellular replication, J. Math. Biol. 38, 241-261, 1999.

[34] T. Li, N. Pintus and G. Viglialoro, Properties of solutions to porous medium problems with different sources and boundary conditions, Z. Angew. Math. Phys. 70 (3), Art. 86, 1-18, 2019. 
[35] X. Li and Z. Wang, Global attractivity for a logistic equation with piecewise constant arguments. Differences and Differ. Eqs., in: Fields Inst. Commmun. 42, 215-222, 2004.

[36] X. Li and D. Zhu, Global asymptotic stability of a kind of nonlinear delay difference equations, Appl. Math.-JCU, Set. B, 17 (2), 178-183, 2002.

[37] X. Li and D. Zhu, Global asymptotic stability in a rational equation, J. Difference Equ. Appl. 9 (9), 833-839, 2003.

[38] X. Li and D. Zhu, Global asymptotic stability for two recursive difference equations, Appl. Math. Comput. 150 (2), 481-492, 2004.

[39] X. Li and D. Zhu, Two rational recursive sequences, Comput. Math. Appl. 47 (10-11), 1487-1494, 2004.

[40] Z. Li and D. Zhu, Global asymptotic stability of a higher order nonlinear difference equation, Appl. Math. Lett. 19 (9), 926-930, 2006.

[41] P. Liu and K. Gopalsamy, Global stability and chaos in a population model with piecewise constant arguments, Appl. Math. Comput. 101, 63-88, 1999.

[42] P. Liu and K. Gopalsamy, Global stability and chaos in a population model with piecewise constant arguments, Appl. Math. Comput. 101 (1), 63-88, 1999.

[43] G. Liu, A. Zhao and J. Yan, Existence and global attractivity of unique positive periodic solution for a LasotaWazewska model, Nonlinear Anal. 64, 1737-1746, 2006.

[44] E. Liz and C. Lois-Prados, A note on the Lasota discrete model for blood cell production, Discrete Contin. Dyn. Syst. Ser. B, 25 (2), 701-713, 2020.

[45] E. Liz, V. Tkachenko and S. Trofmchuk, Global stability in discrete population models with delayed-density dependence, Math. Biosci. 199 (1), 26-37, 2006.

[46] H. Matsunaga, T. Hara and S. Sakata, Global attractivity for a logistic equation with piecewise constant argument, Nonlinear Differential Equations Appl. 8 (1), 45-52, 2001.

[47] Y. Muroya,Persistence, contractivity and global stability in logistic equations with piecewise constant delays, J. Math. Anal. Appl. 270, 602-635, 2002.

[48] Y. Muroya, A sufficient condition on global stability in a logistic equation with piecewise constant arguments, Hokkaido Math. J. 32, 75-83, 2003.

[49] Y. Muroya, Global attractivity for discrete models of nonautonomous logistic equations, Comput. Math. Appl. 53 (7), 1059-1073, 2007.

[50] I. Ozturk and F. Bozkurt, Stability analysis of a population model with piecewise constant arguments, Nonlinear Anal. Real World Appl. 12 (3), 1532-1545, 2011.

[51] F. Qiuxiang and Y. Rong, On the Lasota-Wazewska model with piecewise constant argument, Acta Math. Sci. 26 (2), 371-378, 2006.

[52] S. Rihani, A. Kessab and F. Cherif, Pseudo-almost periodic solutions for a LasotaWazewska model, Electron. J. Differential Equations, 2016, 1-17, 2016.

[53] S.H. Saker, Qualitative analysis of discrete nonlinear delay survival red blood cells model, Nonlinear Anal. Real World Appl. 9, 471-489, 2008.

[54] J. Shao, Pseudo-almost periodic solutions for a LasotaWazewska model with an oscillating death rate, Appl. Math. Lett. 43, 90-95, 2015.

[55] J.W.H. So and J.S. Yu, Global stability in a logistic equation with piecewise constant arguments, Hokkaido Math. J. 24 (2), 269-286, 1995.

[56] G. Stamov and I. Stamova, Impulsive Delayed LasotaWazewska Fractional Models: Global Stability of Integral Manifolds, Mathematics 7 (11), 10-25, 2019.

[57] S. Stevi and D. Tollu, Solvability and semi-cycle analysis of a class of nonlinear systems of difference equations, Math. Methods Appl. Sci. 42 (10), 3579-3615, 2019.

[58] V. Tkachenko and S. Trofimchuk, Global stability in difference equations satisfying the generalized Yorke condition, J. Math. Anal. Appl. 303 (1), 173-187, 2005.

[59] V. Tkachenko and S. Trofimchuk, A global attractivity criterion for nonlinear nonautonomous difference equations, J. Math. Anal. Appl. 322 (2), 901-912, 2006. 
[60] K. Uesugi, Y. Muroya and E. Ishiwata, On the global attractivity for a logistic equation with piecewise constant arguments, J. Math. Anal. Appl. 294 (2), 560-580, 2004.

[61] L. Wang, Qualitative analysis of a predatorprey model with rapid evolution and piecewise constant arguments, Int. J. Biomath. 10 (07), 1750101, 2017.

[62] M. Wazewska-Czyzewska and A. Lasota, Mathematical problems of the dynamics of the red blood cells system, (Polish) Math. Stos. III, 6, 23-40, 1976.

[63] J. Wiener, Generalized Solution of Functional Differential Equations, World Scientific, Singapore, 1993.

[64] S. Xiao, Delay effect in the Lasota Wazewska model with multiple time-varying delays. Int. J. Biomath. 11, 1850013, 2018.

[65] W. Xu and J. Li, Global attractivity of the model for the survival of red blood cells with several delays, Ann. Differential Equations, 14, 357-363, 1998. 